\title{
Philonsorbonne
}

12 | 2018

Année 2017-2018

\section{Spinoza et Sartre : une composition dynamique des singularités}

(Présentation du livre : Spinoza et Sartre. De la politique des singularités à l'éthique de générosité)

\section{Gaye ÇANKAYA EKSEN}

\section{(c) OpenEdition}

\section{Journals}

Édition électronique

URL : https://journals.openedition.org/philonsorbonne/1024

DOI : 10.4000/philonsorbonne. 1024

ISSN : 2270-7336

Éditeur

Publications de la Sorbonne

Édition imprimée

Date de publication : 1 janvier 2018

Pagination : 175-186

ISSN : 1255-183X

Référence électronique

Gaye ÇANKAYA EKSEN, «Spinoza et Sartre : une composition dynamique des singularités »,

Philonsorbonne [En ligne], 12 | 2018, mis en ligne le 21 mars 2018, consulté le 08 juin 2021. URL :

http://journals.openedition.org/philonsorbonne/1024; DOI : https://doi.org/10.4000/philonsorbonne. 1024

(c) Tous droits réservés 


\title{
Spinoza et Sartre : une composition dynamique des singularités
}

\author{
(Présentation du livre : Spinoza et Sartre. De la politique des \\ singularités à l'éthique de générosité)
}

\author{
Gaye ÇANKAYA EKSEN
}

(Université de Galatasaray, Turquie)

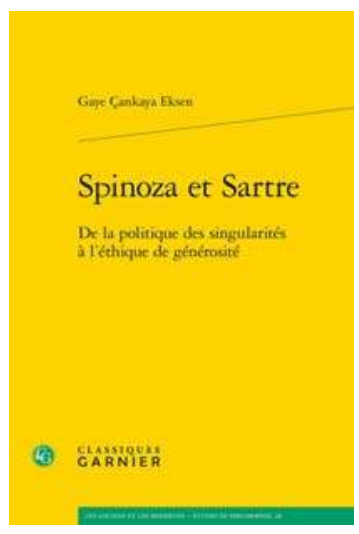

\section{Gaye ÇANKAYA EKSEN}

Spinoza et Sartre. De la politique des singularités à l'éthique des générosités

Au premier abord, les visées et les méthodes philosophiques de Spinoza et de Sartre semblent radicalement différentes. Or, ces différences radicales se trouvent dépassées dès qu'on se penche sur une problématique commune à ces deux philosophes: la production et le maintien de la communauté libre. Une interrogation philosophique sur la question de l'articulation de l'éthique et de la politique nous donnera la possibilité d'évaluer ces philosophes comme les constituants d'une certaine théorie anticontractualiste se fondant spécifiquement sur l'idée de l'émancipation perpétuelle de l'individu dans et par la communauté.

Paris, Classiques Garnier, 2017 Collection «Les Anciens et les Modernes »

$* * *$

Pour présenter le contenu et l'argumentation générale du livre intitulé Spinoza et Sartre. De la politique des singularités à l'éthique de générosité, il faut tout d'abord souligner que ce travail ne tente pas uniquement de confronter les deux philosophes afin d'arriver à une conclusion qui dégagerait la somme des différences et des similitudes entre leurs deux systèmes de pensée. Un travail qui envisagerait de faire usage de deux philosophes manifestement si différents quant à la méthodologie utilisée ou aux points de départ déterminant la construction de leurs systèmes de pensée, courrait le risque de juxtaposer les différences et les similitudes sans 
construire une discussion fructueuse portant directement sur les deux philosophes en question. À première vue, cette confrontation semble rencontrer une difficulté non seulement d'un point de vue chronologique mais aussi ontologique. Le système déterministe de Spinoza et l'existentialisme de Sartre reposent sur des conceptions de l'être et de la liberté qui paraissent incompatibles, de sorte que leurs visions de l'éthique et de la politique peuvent paraître sans commune mesure.

Dans ce travail, nous cherchons à dépasser le risque d'un tel parti-pris de recherche philosophique comparée en plaçant au centre de l'interrogation l'analyse d'une problématique commune par laquelle il devient possible de rapprocher les pensées éthico-politiques de Spinoza et de Sartre d'abord dans un cadre anti-contractualiste : c'est la problématique de la constitution et du maintien d'une société libre. C'est une question d'Alexandre Matheron, l'un des plus importants commentateurs de la philosophie de Spinoza, qui a joué un rôle déterminant dans la construction de cette argumentation éthico-politique. Cette question a déterminé le sens philosophique de la présente recherche, et elle permet de déployer une démarche équilibrée évitant de conférer une position hégémonique à l'un ou à l'autre philosophe et de s'enfermer dans la posture facile de la réfutation de l'un par l'autre. Ainsi, une question de Matheron formulée dans son grand ouvrage intitulé Individu et Communauté chez Spinoza, qui nous invite à nous interroger sur une analogie éventuelle entre la pensée politique de Sartre et la théorie classique contractualiste. Elle permet de mettre en relief les lignes d'argumentation parallèles (et parfois communes) chez les deux philosophes concernant le processus de constitution et de maintien de la société libre. Dans la théorisation de ce processus actif et résistant à toute approche contractualiste, il apparaît qu'une sorte d'articulation de la politique et de l'éthique se pose d'une manière semblable chez les deux philosophes.

La question d'Alexandre Matheron qui se trouve à la source de cette recherche était formulée de la façon suivante : «N'y a-t-il pas, d'ailleurs, une certaine analogie entre la problématique sartrienne du passage de la série au groupe et la problématique classique du passage de l'état de nature à l'état civil ? $\gg^{1}$ Cette interrogation permet de mettre en lumière un principe primordial qui structure l'ensemble de la philosophie de Sartre et qui

1. A. Matheron, Individu et Communauté chez Spinoza, Paris, Les Editions de Minuit, 1988, p. 201. Dans un entretien Alexandre Matheron affirme également que la réflexion concernant la pensée politique de Sartre occupe son esprit. Suite à une remarque faite par Laurent Bove (il signale que Matheron cite Sartre dans la bibliographie du Christ et le Salut des Ignorants et dans Individu et Communauté chez Spinoza), Matheron explique ses références à Sartre: «Dans la bibliographie du Christ, c'était très ponctuel : je disais que, dans la théocratie hébraïque vue par Spinoza, régnait une sorte de fraternité-terreur; et j'avais cité Sartre uniquement à ce propos. Par contre, dans Individu et Communauté, dans mon étude de la théorie spinoziste des passions, je pensais bien davantage à la Critique de la raison dialectique : le passage de la série au groupe, effectivement, ça m'a donné des idées. » (« À propos de Spinoza », Entretien avec Alexandre Matheron réalisé par Laurent Bove et PierreFrançois Moreau, Paris, 20 Juin-Novembre 1997, paru dans Multitudes $\mathrm{n}^{\circ} 3$, novembre 2000.) 
distingue radicalement la philosophie politique du philosophe français de la théorie contractualiste de Hobbes, analysée dans ses grandes lignes pour concrétiser l'approche contractualiste dans l'histoire de la philosophie.

Ce principe est celui de l'irréductibilité et de la particularité spécifique de chaque individu existant chez Sartre. En mettant ce principe au centre de toutes les analyses de la théorie éthico-politique de Sartre, il apparaît d'emblée que le principe d'irréductibilité de chaque individu humain ne renvoie pas à une théorisation ou à une valorisation de la pluralité chaotique des individus isolés mais, tout au contraire, à un autre principe constitutif de la théorie sociale de Sartre : le principe qui consiste à comprendre chaque individu non pas comme indépendant des autres, mais comme une individualité singulière qui peut et doit se comprendre au sein de la pluralité des autres individualités, donc dans une nécessité relationnelle où chacun se constitue comme une manière spécifique d'agir et d'exister. La liberté chez Sartre renvoie nécessairement à une pratique, de la même façon que la notion du conatus chez Spinoza renvoie à un certain processus d'actualisation d'une essence singulière, et ainsi à une pratique existentielle. Le conatus se présente dans la philosophie de Spinoza comme l'auto affirmation nécessaire de chaque chose. Quant à la liberté chez Sartre, elle est définie comme la somme des pratiques de l'existence individuelle humaine ; les deux concepts paraissent donc similaires.

L'accent mis sur ces principes a joué un rôle déterminant dans cette recherche, puisqu'ils se réfèrent également à la philosophie pratique de Spinoza dans laquelle chaque individu humain est élaboré au sein d'une pluralité relationnelle d'individus comme une unique manière d'exister ou de persévérer dans l'être.

Ainsi, la «Première Partie» de Spinoza et Sartre. De la politique des singularités à l'éthique de générosité, plaçant la question de Matheron à l'origine de la recherche, n'envisage pas seulement d'affirmer la méthodologie matheronienne, qui se présente comme une lecture systématique éclairant la pensée de Spinoza dans tous ses aspects métaphysique, éthique et politique ; elle fait en outre le choix explicite de situer la méthodologie et d'évaluer la pensée de Spinoza dans une «tradition » française qui met l'accent sur le terme conatus comme étant le point d'intersection de la métaphysique, de l'éthique et de la politique de Spinoza. Parallèlement à une telle lecture méthodologique de Spinoza, nous proposons une lecture intertextuelle des ouvrages de Sartre en mettant au centre de toutes les analyses éthiques, politiques et ontologiques, la conception de la liberté qui structure sa philosophie comme une philosophie de l'émancipation individuelle et commune.

Dans la première partie de cette recherche, les analyses et les interrogations sur le fonctionnement des termes de sociabilité négative et positive chez Hobbes, Sartre et Spinoza sont successivement exposées. Dans la théorie classique contractualiste, la vision politique se fonde sur une idée de transformation des individus humains en sujets prévisibles et contrôlables 
dans un certain cadre sociopolitique. La constitution du domaine politique correspond à la clarification des points de contrôle des relations humaines par une référence primordiale à la raison.

En définissant la théorie politique de Hobbes dans un tel cadre, il est devenu possible de dire que, dans la théorie contractualiste, l'idée d'identification et de standardisation des individus (par l'usage d'une passion ultime, à savoir la crainte de la mort) joue un rôle très important dans la constitution et dans le maintien de la société politique. La logique du contrat définitif exige des actes raisonnables des individus humains pour s'unir dans une société civile, et par ces actes raisonnables, effectués une fois pour toutes, ceux-ci deviennent des parties identiques devant telle ou telle figure de la souveraineté, laquelle représente la somme des individus raisonnables. Le transfert des droits au souverain par un acte de la raison de chacun est considéré comme le fondement de la légitimité de tous les actes de la figure de souveraineté. Par le transfert définitif des droits, la fonction des individus composant l'état civil est réduite au minimum dans le maintien de la société civile.

À la différence de cette idée de la souveraineté rationnelle et du transfert définitif des droits aux souveraines, dans l'horizon politique où les analyses de Sartre et de Spinoza se déploient parallèlement, le rôle joué par les individus particuliers dans la constitution, le maintien et même dans la dissolution de la société est déterminant, puisque les individus sont considérés non pas comme des sujets identiques devant la figure de la souveraineté absolue, mais comme les manifestations de puissances singulières. Dans une telle perspective qui est commune à Spinoza et à Sartre, chaque individu se présente comme un acteur de l'expérience constructive de la société et du maintien de la société politique. Dans la perspective anti-contractualiste de Spinoza, la souveraineté se définit comme la somme des puissances actives des individus humains, et la démocratie se pose comme «le régime politique par excellence et le plus proche de la liberté que la nature accorde à chacun » (TTP, chap. XVI). Et il est possible d'utiliser une formulation similaire pour la théorie politique sartrienne.

La première partie de notre ouvrage fait également valoir que, s'il est possible de parler d'une certaine rationalité de la politique chez Spinoza et chez Sartre, elle doit se formuler au sein de la conflictualité passionnelle des individus humains. L'analyse effective des individus humains exige en effet une compréhension détaillée de la conflictualité humaine qui se montre à chaque étape de la sociabilité. Cette conflictualité originaire inhérente à tout type de sociabilité implique également une possibilité permanente : c'est la possibilité de résistance de chacun à un autre individu, à son égal, ou à l'ordre constitué dans toutes les formes de sociabilité. Spinoza et Sartre affirment tous deux qu'un ordre civil, quel que soit le régime politique, doit rester conforme aux désirs, aux projets et aux besoins de ses composants; il doit donc se renouveler et se reformuler sans cesse dans différents contextes pour s'adapter aux différents désirs des sujets constituant la société politique. 
Dans ce contexte, on peut également souligner que, pour Spinoza et Sartre, une «société civile juste» doit se former à partir de la libre actualisation des passions; ou du moins, elle doit tenir compte de la possibilité permanente de la libre actualisation des passions de chaque individu. En d'autres termes, un «État juste» doit articuler de manière incessante les passions des individus avec la stratégie d'être ensemble et de vivre dans la concorde commune. Cette tâche d'articulation des passions et de la stratégie d'être ensemble dans la concorde indique également un processus de construction d'une puissance collective qui englobe bien les passions comme l'un des aspects déterminants de la réalité humaine. L'affirmation de toute individualité particulière dans son effectivité singulière marque la conception de la vie politique chez Spinoza et chez Sartre; et la recherche du libre développement de ces individualités dans la pluralité d'individus détermine le fondement et les visées primordiales de leurs théories politiques.

À partir d'une perspective mettant en lumière le rôle irréductible de la singularité propre à chacun dans la constitution et dans le maintien de la vie commune, il est possible de voir un parallélisme entre la théorie politique de Spinoza et la théorie politique de Sartre, dans la mesure où leurs approches s'opposent explicitement à l'idée du passage à la vie civile comme rupture définitive et irréversible. Chez Spinoza, cette opposition apparaît dans les analyses concernant le passage ou bien la transformation de l'état de nature à l'état civil et on trouve également le refus explicite de l'idée d'une rupture entre ces deux états de pluralité humaine dans sa très célèbre Lettre L dans laquelle il écrit les phrases suivantes :

«Vous me demandez quelle différence il y a entre Hobbes et moi quant à la politique : cette différence consiste en ce que je maintiens toujours le droit naturel et je n'accorde dans une cité quelconque de droit de souverain sur les sujets que dans la mesure où, par la puissance, il emporte sur eux; c'est la continuation de l'état de nature. »

Chez Spinoza, l'état civil n'est pas un état rationnel sans passions, ni l'état de nature un état passionnel sans raison. Chez Sartre, une approche semblable apparaît dans les analyses concernant le passage de la série au groupe. Cette approche implique également un refus des formes absolues des sociétés humaines. Selon les deux philosophes, ni les rassemblements négatifs (à savoir la sérialité ou l'état de nature) ni les sociétés positives (à savoir le groupe ou l'état civil) ne peuvent être considérés comme les formes absolues de la pluralité humaine. En effet, chez Spinoza et chez Sartre, ces formes positives et négatives ne peuvent pas non plus être considérées comme des formes absolument opposées. Ces formes sociales se manifestent surtout dans une relation d'entrelacement ou dans un mouvement d'oscillation. Si nous élaborons la structure interindividuelle de la réalité humaine dans un mouvement d'oscillation continue entre la phase négative et la phase positive de la relation interindividuelle, le passage à l'état civil peut être défini comme la prolongation et la stabilisation de la phase positive de l'interaction des puissances. 
Chez Sartre, l'idée qui affirme que les groupes surgissent toujours sur un fond de sérialité et que tous les groupes contiennent en eux-mêmes une possibilité permanente de retourner à la sérialité, indique que les rassemblements humains se réalisent toujours dans un mouvement d'oscillation entre ces deux pôles de la sociabilité. Dans ce mouvement dynamique d'oscillation, un seul facteur déterminant demeure inchangeable : le rôle original de chaque individu fondé sur sa complexion unique, sur sa manière d'exister, donc sur sa liberté originaire. La manière de persévérer dans l'être de chacun ou le mode d'actualisation de son être de chaque individu reste unique dans toutes les formes de sociabilité. Chez Spinoza, pour chaque individu, la stratégie de participation à la vie sociale s'explique à partir du concept du conatus et de droit naturel, et chez Sartre elle s'explique par l'idée de la liberté ontologique de l'individu humain. Une telle perspective prend explicitement ses distances avec des interprétations de Spinoza qui placent la question classique de «l'obéissance» au cœur de sa pensée politique. Dans ce travail, bien au contraire, il nous apparaît que c'est la question de « la liberté », de sa constitution et de sa préservation, qui est au cœur de la philosophie de Spinoza. D'un tel point de vue, on peut alors montrer que la question du désir de vivre en sécurité et celle du désir de vivre ensemble d'une pluralité d'individus libres sont strictement liées l'une à l'autre. Le désir de vivre en sécurité ne se réfère pas à une conception passive de l'obéissance chez Spinoza et chez Sartre, mais il se réfère directement et explicitement au désir de l'actualisation véritable de la liberté particulière de chacun.

Ainsi, dans le cadre du parallèle entre la pensée de Spinoza et celle de Sartre, la conception de la liberté sartrienne doit être élaborée non pas comme une norme généralisée ou un simple libre arbitre, mais comme la somme des intentions, des désirs et des actions, des projets d'un individu, ou comme sa manière d'exister. Il faut souligner que, chez les deux philosophes, l'accent mis sur la particularité de chaque communauté humaine résulte également de cette insistance sur l'irréductibilité de chaque individu singulier et sur les conditions conjoncturelles de la communauté en question.

Dans cette approche politique commune, la théorie politique ne vise pas à découper des grands moments de l'Histoire. La théorie politique s'explique plutôt comme un mouvement de totalisation qui cherche à incorporer tous les types d'expériences à la constitution d'une Histoire. Les deux philosophes partagent donc une perspective qui affirme que chaque société humaine s'exprime par un dynamisme des relations propres à elle-même; et ils partagent également un point de vue selon lequel l'intelligibilité de chaque société se montre à travers son action et sa réflexion commune, lesquelles se manifestent comme des inventions conjoncturelles de cette société. Et les passions des individus déterminent la structuration de la vie sociale et politique dans la mesure où elles résistent à l'ordre civil ou bien le favorisent. 
Pour Sartre et pour Spinoza, la théorie politique ne cherche pas à établir la possibilité d'une société sans conflit. Elle ne cherche pas non plus à théoriser la possibilité d'une vie commune qui se déploierait définitivement en paix. La puissance émancipatrice commune peut naître de telle ou telle conjoncture conflictuelle chez Sartre. De la même façon, chez Spinoza, une situation d'antagonisme, ou même de guerre peut être le point de départ d'un mouvement de libération d'une pluralité d'individus. De ce point de vue, il faudrait admettre qu'aucune analyse politique ne se veut définitive; et, dans la réalité conjoncturelle de chaque société humaine, les principes du vivre ensemble doivent être toujours reconsidérés. Dans le domaine politique, ce que les théories du contrat tentent d'effectuer par la standardisation des individus et par la visée de fixer la vie sociale sans conflit à travers une référence à la raison absolue du souverain semble parallèle à ce que les conduites de la mauvaise foi ou de l'esprit de sérieux tentent d'effectuer dans le domaine éthico-ontologique chez Sartre: résister à l'existence dynamique qui doit se construire par une activité incessante et affirmer l'absoluité des certains règles, situations ou valeurs pour pouvoir dénier l'exigence de la production libre et continue de soi.

Dans les analyses concernant l'affirmation de la particularité de chaque société humaine et de leurs manières uniques de participer à la constitution d'une Histoire, l'évaluation du terme spinoziste d'ingenium par PierreFrançois Moreau joue un rôle déterminant ${ }^{2}$. Pierre-François Moreau insiste sur ce terme qui éclaire la spécificité et la complexion de chaque société humaine dans ses conditions historiques, matérielles et expérientielles spécifiques. Il faut souligner que le terme d' exis $^{3}$ chez Sartre joue un rôle parallèle dans la théorie politique de la Critique de la raison dialectique. L'exis de tel groupe peut être considéré comme la somme des données acquises en particulier en cours d'une expérience originaire, et cette somme exprime le caractère actuel du groupe.

En mettant l'accent sur l'ingenium chez Spinoza et sur l'exis chez Sartre, on peut montrer que chez les deux philosophes, si l'on veut parler d'une communauté libre et unie comme une singularité sociale, il est nécessaire de dégager et d'éclairer une expérience originaire dans laquelle la dynamique relationnelle et active de cette communauté surgit. Il s'agit donc ici d'un rapprochement des théories sociales de Spinoza et de Sartre, puisqu'ils tentent de comprendre la société civile non pas à partir d'une décision rationnelle et hypothétique des individus, mais à partir d'une expérience commune, concrète et émancipatrice qui va atteindre à une certaine définition de la complexion interindividuelle et sociale. Notons que si l'ingenium et le caractère d'une singularité commune se réfèrent particulièrement à l'expérience passée et aux conditions matérielles d'une communauté, ils se présentent comme des termes empreints de passivité. Ces

2. P.-F. Moreau, Spinoza, L'expérience et l'éternité, Paris, PUF, 1994.

3. J.-P. Sartre, Critique de la raison dialectique, Tome I, Théorie des ensembles pratiques, Paris, NRF, Gallimard, 1960, p. 287, 307, 362, 374, 386, 390, 414, 456. 
conceptions semblent présenter un risque : celui de fixer et de réduire un peuple ou un groupe à un caractère défini une fois pour toutes par une expérience originaire. Mais Spinoza et Sartre résistent parallèlement à une telle approche absolutisant un seul moment du processus social d'une pluralité humaine. Ces conceptions semblables chez les deux philosophes servent à affirmer l'originalité de chaque communauté, et elles doivent être comprises comme la définition de telle ou telle communauté humaine qui se renouvelle sans cesse dans l'interaction actuelle des individus participant à cette communauté. Les termes d'ingenium et d'exis sont utilisés pour expliquer l'intelligibilité de l'être ensemble des individus humains à travers l'analyse de la logique de la composition, de la permanence et de la décomposition de sociétés dans certaines conditions.

Dans ce contexte, en soulignant l'aspect expérientiel de la constitution de la société civile, on montre également que Spinoza comme Sartre affirment explicitement la simultanéité de la libération individuelle et de la libération collective. Les deux philosophes expliquent l'expérience libératrice à partir d'un état de crise et d'urgence dans lequel une certaine structure nécessaire fonctionne dans la spontanéité des actions interindividuelles. Le passage de la multitude soumise à la multitude libre chez Spinoza, et le passage de la série au groupe en fusion chez Sartre sont expliqués, d'une manière parallèle, dans une logique spontanée des actions individuelles qui se lient les unes aux autres afin d'arriver au même but.

Par une lecture détaillée de la Critique de la raison dialectique de Sartre, il est très intéressant de voir que Sartre explique les dynamiques du surgissement d'une action spontanée dans le cas d'urgence à partir de l'idée de contagion. Dans le processus de dissolution de la sérialité et du surgissement du groupe en fusion, les individus passent par une étape irréfléchie, et la contagion sérielle se manifeste comme un ordre inscrit dans l'existence de chaque individu humain; à partir de cette quasi-unification contagionnelle de l'action, la pluralité des individus peut alors être comprise comme une action unifiée. Dans cette spontanéité, Sartre voit une nécessité structurelle qui conduit les individus humains à établir toujours des liens de réciprocité positive. Notre travail montre que l'explication du passage de la multitude soumise à la multitude libre se réfère à une logique semblable mais beaucoup plus détaillée - qui s'explique par l'imitation des affects ou par la contagion des affects chez Spinoza. Ici, il est important de voir que la pensée politique de Spinoza se fonde sur une théorie pertinente des affects par laquelle on comprend les étapes et le fonctionnement de cette contagion affective spontanée qui détermine l'action originaire et émancipatrice. La référence à l'analyse d'Alexandre Matheron au sujet de l'affect de l'indignation permet également de souligner que l'enchaînement très précis des affects détermine ce processus spontané du surgissement d'une multitude libre. Mais, à la différence de Spinoza, il n'est pas possible de mettre en lumière chez Sartre les étapes ou les détails de cette logique contagionnelle. Sartre tente d'expliquer la possibilité du fonctionnement contagionnel de l'activité d'urgence par la force des causes extérieures qui structure 
immédiatement une activité commune des semblables. Le passage de la réciprocité négative à la réciprocité positive chez Sartre s'explique par une réaction spontanée et contagionnelle à un appel d'urgence'. C'est l'unification synthétique du divers. Cette terminologie sartrienne de «la réciprocité négative et la réciprocité positive » est empruntée par Matheron dans ses analyses de la transformation de la multitude soumise en multitude libre chez Spinoza. Chez Spinoza comme chez Sartre, nous pouvons définir le passage d'une forme négative de la société (de la série ou de la multitude soumise) à une forme positive (au groupe ou à la multitude libre) : il s'agit d'une création conjoncturelle imposée par les conditions particulières du cas spécifique dans lequel les individus se transforment spontanément en acteurs. L'expérience commune devient alors la seule unité réelle des individus. Même si la logique de contagion apparait plus explicitement dans la pensée spinoziste par l'analyse de l'affect de l'indignation et ne se trouve pas dans la pensée de Sartre, les deux auteurs ont une manière semblable d'expliquer le passage à la réciprocité positive.

Même si Sartre ne détaille pas cette explication, on peut inférer du parallélisme mis en lumière entre ses analyses et celles de Spinoza le schéma des quatre étapes du surgissement de la société libre, communes aux deux philosophes ${ }^{5}$ :

1) L'étape du surgissement d'un danger qui menace l'individu (et les autres individus).

2) L'étape de la perception de ce danger comme un danger commun. C'est l'étape du mouvement contagionnel et spontané (donc, l'étape irréfléchie où émerge le rassemblement des individus semblables).

3) L'étape de la prise de conscience de la pluralité des mouvements individuels comme action commune (c'est l'étape d'activité et de considération simultanée des autres individus en tant que mêmes ou en tant qu'individus semblables).

4) L'étape de la réflexion sur l'expérience commune et de l'organisation de la communauté pour le maintien des acquis positifs de l'expérience commune.

Dans la deuxième partie de Spinoza et Sartre. De la politique des singularités à l'éthique de générosité, la quatrième étape de ce schéma partagé par Spinoza et Sartre est particulièrement analysée comme le moment d'expression de l'articulation de la politique et de l'éthique. Le passage à l'organisation de la société commune est analysé comme constitution de la stabilité d'un certain degré de perfectionnement (et donc de la liberté) obtenu dans l'expérience commune originaire. Dans cette étape de sociabilité, un processus de contemplation active de soi, ou encore une

4. J.-P. Sartre, Critique de la raison dialectique, Tome I, Théorie des ensembles pratiques, Paris, NRF, Gallimard, 1960, p. 471-472.

5. Mais il faut signaler que ces étapes ne sont pas schématisées comme telles de façon explicite dans leur pensée. 
prise de conscience de soi, devient central chez les deux philosophes. Ainsi, l'étape de la constitution de la concorde et de la paix dans une communauté libre contient chez eux une certaine activité réflexive qui transforme la relation de l'individu à la fois avec lui-même et avec les autres individus. À ce moment particulier de l'établissement de la concorde dans la pluralité des individus libres, Spinoza et Sartre adoptent des stratégies semblables pour articuler la politique et l'éthique : ils affirment que le maintien de la liberté commune et individuelle ne dépend pas uniquement des organisations pratiques des institutions d'un gouvernement, mais qu'il dépend en même temps de la réflexion éthique et active de chaque individu conscient que la liberté ne peut être construite que dans la pluralité des individus.

Chez Sartre, la réflexion pure qui exprime un rapport authentique à soi et qui se manifeste comme une activité réflexive aboutit à la générosité qui est considérée comme l'assomption et la concrétisation véritable de la liberté. À partir de la prise de conscience de la structure plurielle de la liberté, la générosité devient le principe primordial des relations affirmatives interindividuelles. Parallèlement, chez Spinoza, la notion de "force d'âme » se réfère directement, par ses deux modes inséparables, à savoir la fermeté et la générosité, au principe ou à la stratégie de vivre librement dans la pluralité des singularités. Chez Spinoza et chez Sartre, la véritable affirmation de soi et l'affirmation d'autrui coïncident par là dans le domaine éthico-politique. Mais à ce point de l'analyse, on peut légitimement se demander si on a affaire à une simple homonymie entre la générosité sartrienne et spinoziste ou bien si les deux concepts ont effectivement des propriétés communes. La générosité conçue comme don de soi est présentée par Sartre comme passion dans les Cahiers pour une Morale. On peut se demander, comme Chantal Jaquet l'a souligné dans la Préface, si elle a une commune mesure avec la générosité spinoziste qui est un affect actif et se présente non comme une passion mais comme une action. Ici, il faut tout d'abord remarquer que l'expression de «passion» n'a pas un poids philosophique chez Sartre comme elle l'a dans la philosophie spinoziste. Mais, une telle explication ne peut être suffisante. Pour pouvoir donner une réponse plus convaincante, il faut rappeler que la générosité est une valeur première ressortissant à l'ordre du pour-soi. Cette valeur s'incarne dans toute action créatrice authentique (c'est-à-dire toute action qui se fonde sur la réflexion pure et non-complice) en tant qu'elle donne aux autres le monde à voir, à comprendre et finalement à transformer. Le résultat des comportements de générosité est une grande transformation ontologico-éthique: cette transformation est si radicale qu'elle signifie la coïncidence de l'aspect pour-soi de l'individu avec son aspect pour-autrui. C'est par la voie d'une telle transformation que Sartre tente de résoudre le problème de l'intersubjectivité qui apparaît dans $L$ 'Etre et le Néant. La relation de la générosité à la conception de la réflexion pure et l'authenticité chez Sartre implique que la générosité doit être comprise au delà d'une simple passion au sens classique du terme. Car elle est effectivement un acte d'exister et d'agir par l'assomption d'une structure véritable de la réalité humaine qui doit se construire toujours-déjà dans et 
par la pluralité d'individus. Mais Sartre ayant utilisé littéralement le terme de «passion » dans les Cahiers pour une morale, une telle explication est nécessaire pour mieux comprendre le contenu du terme. Il faut noter que la possibilité de la coïncidence du principe de conservation de soi et du principe d'alliance découle de l'idée que la liberté n'exprime qu'un processus continu de modification des relations d'interdépendance dans la pluralité des singularités.

Dans la deuxième partie de ce travail, la définition sartrienne de la générosité est analysée comme un principe réflexif de libération, dans le domaine concret de la pluralité des existences interdépendantes. Chez Sartre, le passage à la réflexion pure, qui implique une assomption ontologique de la structure véritable de l'existence humaine, englobe la prise de conscience de soi dans la totalité de l'existence. Ce positionnement adéquat de soi dans la totalité de l'existence donne à l'individu la possibilité de modifier ses propres possibles en vue d'affirmer son existence en même temps que celle d'autrui.

Dans la réflexion pure sartrienne, qui se fonde sur la compréhension et l'assomption de la structure ontologique de l'individu humain, et dans la force d'âme spinoziste, qui se fonde sur la connaissance adéquate du fonctionnement de l'existence, il s'agit d'une libération à la fois cognitive et praxique. La théorie politique et l'éthique fonctionnent chez Sartre et chez Spinoza comme des théories pratiques qui visent à organiser les relations interhumaines en vue de favoriser la liberté individuelle de chacun comme condition de possibilité d'une liberté commune véritable. Dans ce cadre, le risque perpétuel de la conflictualité ne se pose pas comme un échec qui cause nécessairement la décomposition de la communauté libre, mais plutôt comme la base des nouvelles praxis communes qui structurent et modifient la communauté au cours d'un renouvellement continu de l'actualité humaine.

L'élucidation des théories politiques de Spinoza et de Sartre autour d'une discussion sur la production et le maintien de la communauté libre permet de définir la politique selon deux étapes complémentaires ; l'une est l'étape spontanée ou préréflexive du surgissement de l'action commune qui se manifeste dans les états de crise ou d'urgence, et l'autre est l'étape de l'organisation et de la stabilisation réflexive.

Dans la première étape, les individus agissent selon une logique de contagion spontanée déterminée par les causes extérieures menaçant l'existence d'une certaine pluralité d'individus. À cette étape, l'unité négative des individus humains se transforme en une unité positive dans la structure nécessaire de l'action commune qui vise à une fin commune.

Dans la deuxième étape de la politique, l'action commune s'accomplit, et l'expérience commune et ses conséquences deviennent le contenu de la donnée commune de la société en question. Loin d'être un processus de fixation des conditions données suivant l'action commune, cette étape s'explique comme la continuation réflexive d'un mouvement émancipateur. Dans cette étape 
réflexive de la politique, les questions de régime, d'institutionnalisation et de sécurité deviennent centrales et la réflexion éthico-ontologique s'effectue comme la base des relations interindividuelles.

Dans la conclusion de ce travail, la définition de l'articulation entre la politique et l'éthique dans l'étape réflexive de la politique aboutit à définir l'éthique, chez Sartre et chez Spinoza, en tant que domaine pratique qui s'exprime toujours par telle ou telle prise de conscience devant le fonctionnement de la réalité humaine interindividuelle. L'action éthique (qui se concrétise par la force d'âme chez Spinoza ou par la réflexion pure et la générosité chez Sartre) exige par conséquent une pratique réflexive véritable qui se présente comme une prise de conscience de la structure ontologique de la réalité humaine. Il faut noter qu'une telle prise de conscience éthique peut se manifester non pas dans les états de crise et d'urgence, mais plutôt dans une communauté libre qui est en train de s'organiser ou qui est déjà organisée.

La contemplation active de soi et des autres devient alors possible en particulier dans une communauté qui a dépassé la phase négative de la pluralité humaine dans et par une action libératrice. Cependant, dans une communauté libre bien organisée, la réflexion éthique de chacun ne se pose pas comme une nécessité, mais plutôt comme une possibilité favorisant le fonctionnement libérateur des relations interindividuelles. C'est pour cela que ni Spinoza ni Sartre n'expliquent la société politique comme une communauté de sages ou d'individus toujours rationnels ou émancipés une fois pour toutes. Une communauté libre et organisée contient toujours des conflictualités interindividuelles et la politique doit créer sans cesse des solutions conjoncturelles pour les conflits récurrents. La réflexion et l'action éthique, qui visent à comprendre et à modifier le fonctionnement des relations humaines pour favoriser la liberté et la sécurité à la fois commune et individuelle, s'articulent à l'activité politique perpétuelle en tant que domaine de production des relations favorisant le perfectionnement commun et individuel. En dernière analyse, comme les deux philosophes l'affirment de manière explicite, le contexte véritable d'une problématisation éthique est une société politique.

Dans un tel contexte, l'éthique s'explique par les applications concrètes de la prise de conscience libératrice et éthico-ontologique des individus humains dans la société politique. Les fins primordiales de l'éthique et de la politique coïncident dans un but commun, qui est celui de la production continue du mouvement libérateur à travers la modification adéquate des relations interhumaines. En conséquence, la question de la liberté apparaît nécessairement comme une question politique, éthique et ontologique, comme c'est le cas chez nos deux philosophes. 